Aberdeen in 1964-65 when interviewing was carried out. From this sample of 2500,300 possibly asthmatic children were medically examined and their parent or guardian interviewed by a paediatrician. 121 children were confirmed to be asthmatic. There were 84 boys and 37 girls: 16 of the 20 severe cases were boys. Age of onset of asthma showed that $98(80 \%)$ had experienced their first attack by the age of 5 .

There was a family history of asthma among first degree relatives in $64(53 \%)$, of eczema in $14(12 \%)$, and of allergic rhinitis in $21(17 \%)$. The asthmatic children themselves showed other allergic manifestations: 35 $(29 \%)$ had had infantile eczema, $27(22 \%)$ had had flexural eczema, and $49(37 \%)$ had had allergic rhinitis.

Review of therapy revealed that only one of the asthmatic children had received steroids before the survey.

The group of 121 were graded according to severitymild (50), moderate (51), and severe (20). Allocation was made on the basis of history and examination. Subsequent evaluation of pulmonary function tests showed good correlation with this grading.

Heights and weights of children in all grades tended to be below the mean, but those in the severe grade were at or less than $2 \mathrm{SD}$ below the mean.

Sociological data obtained and evaluated revealed that there were significantly more of the severe asthmatics in the semi- and unskilled manual classes. The severe asthmatics tended to occur in families of 4 or more, regardless of social class. The IQ score at $7+$ years showed that all asthmatics tended to score higher than the rest of the population. This was particularly apparent in the semi- and unskilled manual classes where the mean IQ of the asthmatics was 109 and that of the population 102 .

The over-all prevalence of asthma in this sample of Aberdeen schoolchildren was $4 \cdot 8 \%$.

\section{Arterial Blood Gas Tensions and $p H$ in Acute Asthma in Childhood}

\author{
H. Simpson \\ Department of Child Life and Health, \\ University of Edinburgh
}

The arterial blood gas tensions and $p \mathrm{H}$ in 21 children studied during 24 acute attacks of asthma were reported. All were hypoxic on admission to hospital, and in 10 there was evidence of $\mathrm{Co}_{2}$ retention. Cyanosis, invariably present when the arterial oxygen saturation was below $85 \%$, and restlessness in patients breathing air, were the most reliable indices of the severity of hypoxia. There were no reliable clinical guides to the $\mathrm{PCO}_{2}$ level. Conventional oxygen therapy in tents (25-40\%) did not always relieve hypoxia, and in 3 cases the administration of oxygen at a concentration of $40 \%$ or over failed to produce a normal arterial oxygen tension. Uncontrolled oxygen therapy could aggravate respiratory acidosis, and 2 children developed $\mathrm{CO}_{2}$ narcosis while breathing oxygen. The necessity for blood gas measurements in the management of severe acute asthma in childhood was emphasized.

\section{Some Biochemical Aspects of Intrauterine Growth Retardation}

\author{
Forrester Cockburn \\ Department of Child Life and Health, \\ University of Edinburgh
}

A group of 9 infants with birthweight below the 10th centile for gestational age, born to mothers with preeclamptic toxaemia and a low urinary excretion of oestriol, were studied at birth and daily during the first week of life.

Samples of blood were obtained at caesarean section from maternal vein, umbilical vein, and umbilical artery. At 24-hour intervals capillary blood samples were obtained 2-2 $\frac{1}{2}$ hours after standard evaporated milk feeds. Values obtained from a group of 15 normally grown infants delivered by caesarean section were used as controls.

Haematocrit and $p \mathrm{H}$ values in maternal and fetal samples of control and low birthweight (LBW) groups were comparable at birth. During the first 4 days after birth the haematocrit was significantly higher in the LBW infants. Capillary $p \mathrm{H}$ values were lower in the LBW group throughout the first week. A mean maternal vein-umbilical vein difference of $24 \mathrm{mg}$. glucose $/ 100 \mathrm{ml}$. plasma was found in the LBW group compared with $10 \mathrm{mg} . / 100 \mathrm{ml}$. plasma in the normal. The umbilical venous and arterial glucose concentrations in the LBW infants were significantly below those of the controls and remained lower throughout the first week. Total plasma protein concentrations of the LBW group were significantly below normal in maternal as well as in all the perinatal samples. There were no gross differences in umbilical vein plasma amino acid concentrations apart from a higher taurine concentration in the LBW. samples. The over-all ratio of umbilical vein/maternal vein plasma amino acid concentration was reduced from 1.9 (control) to 1.5 (LBW) due to an increase in the toxaemic mothers' amino acid concentrations towards the non-pregnant values.

Plasma calcium was significantly lower and inorganic phosphorus higher in the umbilical vein plasma of the LBW infants. Magnesium values were comparable throughout. Concentrations of copper were higher in the plasma of the toxaemic mothers but fetal concentrations were normal.

The relevance of these findings in relation to placental function and the subsequent development of the infants was discussed.

\section{Folic Acid Trends and Prophylaxis in Prevention of Megaloblastic Anaemia of Infants of Low Birthweight}

T. F. P. Mackintosh, M. K. Strelling,

C. H. M. Walker, and H. B. Goodall

Departments of Child Health and Pathology,

University of Dundee, and Dundee Royal Infirmary

In the first phase of this study the trends in whole 
blood folate in the first 9 months of life were established in 76 infants; 54 were of low birthweight ( $2500 \mathrm{~g}$. or less) and 22 were mature ( $3000 \mathrm{~g}$. or more delivered between 39 and 41 weeks' gestation). The coincident changes in $\mathrm{Hb}$, reticulocyte count, and buffy coat morphology were also studied.

The mean whole blood folate at birth was $339 \mathrm{ng} . / \mathrm{ml}$., and over the first 5-10 weeks there was a consistent fall to a mean of $76 \mathrm{ng} . / \mathrm{ml}$.

The lowest levels (mean $<20 \mathrm{ng}$. $/ \mathrm{ml}$.) were found in 11 low birthweight infants who either developed frank megaloblastic anaemia ( 7 infants) or who showed transitional changes (4 infants).

In the second phase 20 infants weighing less than 2000 g. were studied. Alternate cases were given 100 $\mu \mathrm{g}$. folic acid daily from the third week of life and the effects on whole blood folate and cytology were assessed in 10 infants given prophylaxis and 10 controls at 3, 6, 8, 10 , and 12 weeks.

In the controls the whole blood folate levels declined steadily, and at 12 weeks the mean was $68 \mathrm{ng} . / \mathrm{ml}$. In contrast, levels in the babies receiving prophylaxis rose steadily (mean at 12 weeks $137.5 \mathrm{ng} . / \mathrm{ml}$.). This difference was significant $(\mathrm{p}<0.001)$.

Only 2 infants developed megaloblastic anaemia, one in the control and one in the treatment group. The over-all prevalence of megaloblastic anaemia in the second phase of the study was much lower than that found in the first phase, and is probably due to the fact that all mothers now receive prophylactic folic acid in pregnancy.

$100 \mu \mathrm{g}$. folic acid daily will eliminate folic acid deficiency as a cause of megaloblastic anaemia in low weight infants, but sporadic cases may still occur due to other causes.

\section{'Germ-free' Patient Care \\ Ron Barnes \\ Institute of Child Health, London}

'Germ-free' animals have been described for more than 50 years. In recent years certain 'germ-free' animal techniques have been employed in various fields of human medicine. We have now developed a maintenance isolator modelled upon our existing germ-free animal isolation units. The microbiological integrity of this human isolation unit was first confirmed by the successful maintenance of 'germ-free' mice. Now the unit has been used for the care of a 'germ-free' baby. With the possibility of an inherent immunological defect the infant was delivered and transferred to the maintenance isolator for investigation. A film was shown of this complete procedure, beginning with the germ-free animal studies that led to the delivery and maintenance of the first recorded 'germ-free' baby. 